\title{
Electron injection and heating via turbulent magnetic reconnection at nonrelativistic shocks of young supernova remnants
}

\author{
A. Bohdan*, J. Niemiec, O. Kobzar, \\ Instytut Fizyki Jądrowej PAN, ul. Radzikowskiego 152, 31-342 Kraków, Poland \\ E-mail: artem.bohdan@ifj.edu.pl
}

M. Pohl

Institute of Physics and Astronomy, University of Potsdam, Karl-Liebknecht-Strasse 24/25,

14476 Potsdam, Germany

DESY, Platanenallee 6, 15738 Zeuthen, Germany

E-mail: marpohl@uni-potsdam.de

\begin{abstract}
Electron injection constitutes a central unresolved problem for diffusive shock acceleration. Here we study perpendicular nonrelativistic collisionless shocks in a regime of high Mach numbers, as appropriate for young supernova remnants. We use high-resolution large-scale two-dimensional fully kinetic particle-in-cell simulations that sample a representative portion of the turbulent shock front and account for time-dependent effects of cyclic shock reformation. The microphysics of perpendicular shocks in weakly magnetized plasmas is governed by ion reflection from the shock that leads to the formation of magnetic filaments in the shock ramp resulting from ion-beam filamentation instabilities, and also electrostatic Buneman modes in the shock foot. The latter can provide electron injection through shock-surfing acceleration. Recent studies show that additional electron acceleration can also occur due to spontaneous magnetic reconnection triggered within magnetic filaments in the turbulent shock transition. We study conditions allowing for efficient electron pre-acceleration by this process. We demonstrate a dependence of the magneticreconnection rate on the temperature of the inflowing plasma and also on numerical parameters of the simulations, such as the ion-to-electron mass ratio and the configuration of the average magnetic field with respect to the simulation plane. We discuss the resulting electron spectra and the relevance of our results to the physics of fully three-dimensional systems.
\end{abstract}

35th International Cosmic Ray Conference - ICRC2017

10-20 July, 2017

Bexco, Busan, Korea

* Speaker. 


\section{Introduction}

Acceleration of charged particles is a key topic in astrophysical research. High-energy particles are found at astrophysical and interplanetary shocks, and collisionless nonrelativistic shocks of supernova remnants (SNRs) are widely believed to be the sources of galactic cosmic rays (CRs) with energies up to the knee at $\sim 10^{15} \mathrm{eV}$. Diffusive shock-acceleration (DSA) is a widely accepted acceleration mechanism responsible for energetic particles at supernova shocks [1]. Particle injection into DSA is a still unresolved question, in particular for electrons. Recently it was demonstrated that spontaneous turbulent magnetic reconnection occurs at perpendicular highMach-number shocks [3], providing a new channel for electron energization in addition to the shock surfing acceleration (SSA) $[4,5,6]$.

The characteristic length scales of electrons should be resolved in studies of the microphysics of electron acceleration, and thus we conduct particle-in-cell (PIC) simulations of perpendicular non-relativistic high-Mach-number shocks in 2D3V configuration meaning that we follow two spatial coordinates and all three components of velocity and electromagnetic fields. In contrast to hybrid simulations, PIC simulations follow electron trajectories as well as the ion dynamics.

We concentrate on a parameter regime typical of young SNRs and follow the theoretical treatment described in [5] to provide appropriate conditions at the shock foot for electron preacceleration via SSA. The influence of turbulent magnetic reconnection on the efficiency of electron acceleration is scrutinized.

\section{Simulation setup}

In our simulations, two counter-streaming electron-ion plasma slabs collide with each other and form a system of two shocks propagating in opposite directions (see Fig. 1). The simulation box is placed in the $x-y$ plane with open $x$-boundaries and periodic boundary conditions in the $y$-direction. Plasma is continuously injected at both sides of the simulation box. The injected plasma carries a uniform magnetic field, $\vec{B}_{0}$, which is perpendicular to the plasma flow (in the $y-z$ plane) and forms an angle $\varphi$ with the $y$-axis. As the magnetic field is assumed to be frozen into the

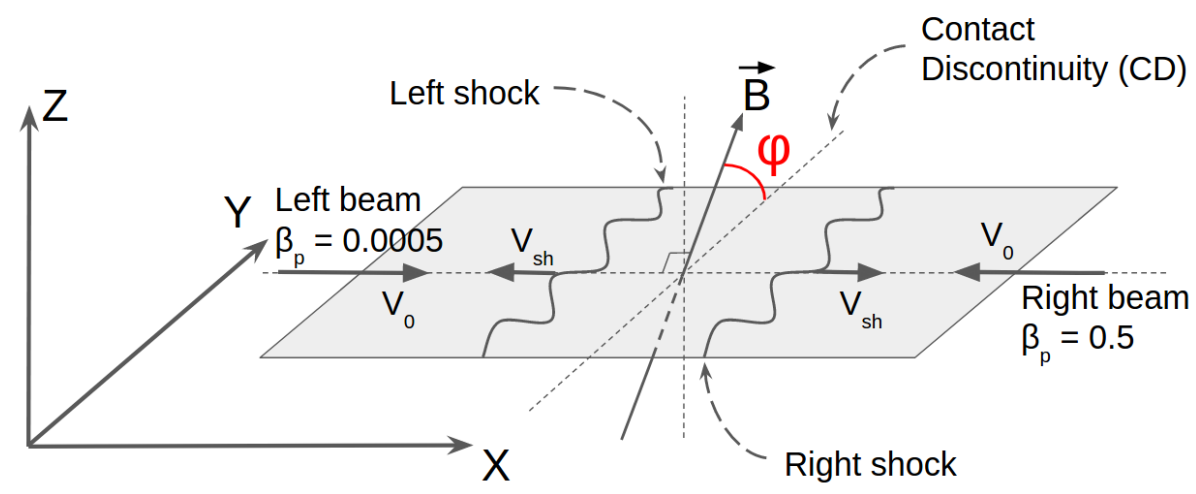

Figure 1: Illustration of the simulation setup. 


\begin{tabular}{ccccccccccc}
\hline \hline Run & $\varphi$ & $m_{i} / m_{e}$ & $\beta_{p}$ & $L_{y}\left[\lambda_{s i}\right]$ & $T\left[\Omega_{i}^{-1}\right]$ & $M_{A}$ & $M_{s}$ & VGR & AD & NTEF [\%] \\
\hline \hline A1 & $0^{\circ}$ & 50 & 0.0005 & 6.3 & 8.3 & 22.6 & 1096 & 0.2 & 1.13 & $0.1 \pm 0.1$ \\
$\mathrm{~A} 2$ & $0^{\circ}$ & 50 & 0.5 & 6.3 & 8.3 & 22.6 & 35 & 0.2 & 1.01 & $0.5 \pm 0.2$ \\
\hline $\mathrm{B} 1$ & $0^{\circ}$ & 100 & 0.0005 & 24 & 8.1 & 31.8 & 1550 & 0.7 & 0.99 & $0.2 \pm 0.1$ \\
$\mathrm{~B} 2$ & $0^{\circ}$ & 100 & 0.5 & 24 & 8.1 & 31.8 & 49 & 0.9 & 1.01 & $0.7 \pm 0.1$ \\
\hline $\mathrm{C} 1$ & $0^{\circ}$ & 200 & 0.0005 & 11.9 & 6.3 & 44.9 & 2192 & 1.9 & 1.03 & $0.2 \pm 0.1$ \\
$\mathrm{C} 2$ & $0^{\circ}$ & 200 & 0.5 & 11.9 & 6.3 & 44.9 & 69 & 2.3 & 1.04 & $0.5 \pm 0.1$ \\
\hline $\mathrm{D} 1$ & $0^{\circ}$ & 400 & 0.0005 & 8.2 & 4.9 & 68.7 & 3363 & 4.4 & 1.01 & $0.4 \pm 0.1$ \\
$\mathrm{D} 2$ & $0^{\circ}$ & 400 & 0.5 & 8.2 & 4.9 & 68.7 & 106 & 5.8 & 1.04 & $0.5 \pm 0.1$ \\
\hline $\mathrm{E} 1$ & $45^{\circ}$ & 50 & 0.0005 & 6.3 & 8.3 & 22.6 & 1096 & 0.3 & 0.82 & $0.2 \pm 0.1$ \\
$\mathrm{E} 2$ & $45^{\circ}$ & 50 & 0.5 & 6.3 & 8.3 & 22.6 & 35 & 0.3 & 0.80 & $0.4 \pm 0.1$ \\
\hline $\mathrm{F} 1$ & $45^{\circ}$ & 100 & 0.0005 & 24 & 8.1 & 31.8 & 1550 & 1.4 & 0.81 & $0.2 \pm 0.1$ \\
$\mathrm{~F} 2$ & $45^{\circ}$ & 100 & 0.5 & 24 & 8.1 & 31.8 & 49 & 1.5 & 0.83 & $0.5 \pm 0.1$ \\
\hline
\end{tabular}

Table 1: Parameters of the simulations and derived shock and downstream properties. $L_{y}-$ transverse size of the simulation box in units of the ion skin depth, $\lambda_{\mathrm{si}}, T$ - simulation time in units of inverse ion cyclotron frequency, $M_{A}, M_{s}$ - Alfvénic and sonic Mach numbers of the shock in the upstream frame, respectively, VGR - vortex generation rate, AD - mean vortex density, and NTEF - nonthermal electron fraction.

moving plasma, a motional electric field $\mathbf{E}=-\mathbf{v} \times \mathbf{B}$ is also initialized in the left and right beam, with $\mathbf{v}=\mathbf{v}_{\mathrm{L}}$ or $\mathbf{v}=\mathbf{v}_{\mathrm{R}}$, respectively. A special setup [4] was used to avoid the artificial antenna effect during the initial collision of the two plasma slabs.

The counter-streaming plasma beams move with equal absolute velocities, $v_{\mathrm{L}}=0.2 c=v_{\mathrm{R}}$, so they collide with a relative velocity of $v_{\text {rel }} \simeq 0.38 c$, where $c$ is the speed of light. The densities of colliding beams are equal, while their temperatures are different. Specifically, we set the plasma beta (the ratio of the plasma pressure to the magnetic pressure) in the left slab to $\beta_{\mathrm{p}, \mathrm{L}}=0.0005$ and in the right slab to $\beta_{\mathrm{p}, \mathrm{R}}=0.5$. So for a single run the only different parameter of the two shocks is the temperature, while all the other parameters remain the same. We perform six numerical experiments with two values of the angle $\varphi$, namely in-plane magnetic field $\varphi=0^{o}$ (runs $\mathrm{A}^{*}, \mathrm{~B}^{*}$, $\mathrm{C}^{*}$, and $\mathrm{D}^{*}$ ), and $\varphi=45^{\circ}$ magnetic field configuration (runs $\mathrm{E}^{*}$ and $\mathrm{F}^{*}$ ). Run-specific parameters are listed in Table 1. Note that the digits in the run designations refer to the left $\left(\beta_{\mathrm{p}, \mathrm{L}}=0.0005\right.$, runs $* 1)$ and right $\left(\beta_{\mathrm{p}, \mathrm{R}}=0.5\right.$, runs $\left.* 2\right)$ shock, respectively.

A number of values of the ion-to-electron mass ratios, $m_{i} / m_{e}$, are considered to investigate the influence of this parameter on the structure of magnetic reconnection regions. The Alfvénic Mach numbers of the shocks depend on $m_{i} / m_{e}$, because we chose to maintain the trapping condition of the Buneman instability (see formula 8 of [5] or equation 4 of [4]) while keeping the plasma beta constant. Thus in all cases we should expect efficient electron acceleration. All important shock parameters and some derived properties of magnetic-reconnection events are listed in Table 1.

The code used in this study is a 2D3V-adapted and modified version of the relativistic electromagnetic particle code TRISTAN with Message Passing Interface-based parallelization [7, 8]. The 
numerical model is essentially the same as that used in [4]. A notable addition is the possibility to follow individual selected particle trajectories, which allows us to study particle acceleration processes in detail.

\section{Simulation results}

\subsection{Spontaneous magnetic reconnection}

The structure of high-Mach-number nonrelativistic perpendicular shocks is discussed in a number of articles [5, 4, 9]. The shock transition consists of a foot, a ramp, an overshoot and the downstream region. Here we concentrate our attention on the ramp or the Weibel instability region. The Weibel-type filamentation instability arises from interaction between shock reflected ions and upstream plasma ions. These filaments are associated with current filaments and filamentary magnetic fields $[3,4,9]$.

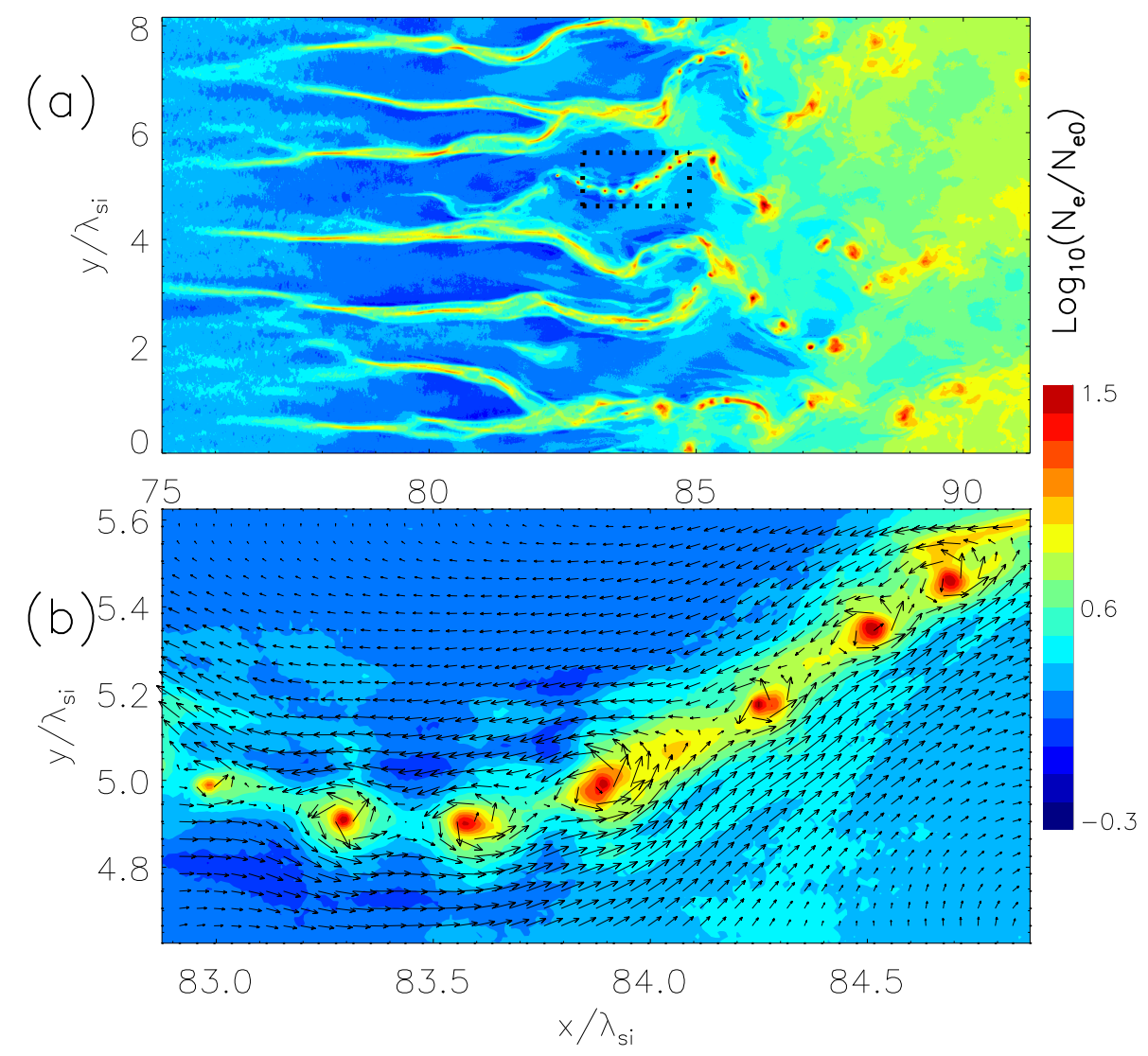

Figure 2: The ramp region for run D1 (panel (a)) at time $T=2.7 \Omega^{-1}$. The region marked with dotted lines in panel (a) is shown enlarged in panel (b). It harbors a chain of magnetic islands. The density is presented in a logarithmic scale and normalized to the upstream density. Arrows show the in-plane (x-y) component of the magnetic field.

As was shown in [3] for the in-plane magnetic-field configuration, magnetic filaments can trigger spontaneous turbulent magnetic reconnection in the shock ramp. We observe magnetic reconnection events not only for the in-plane configuration, but also for the $\varphi=45^{\circ}$ configuration. 


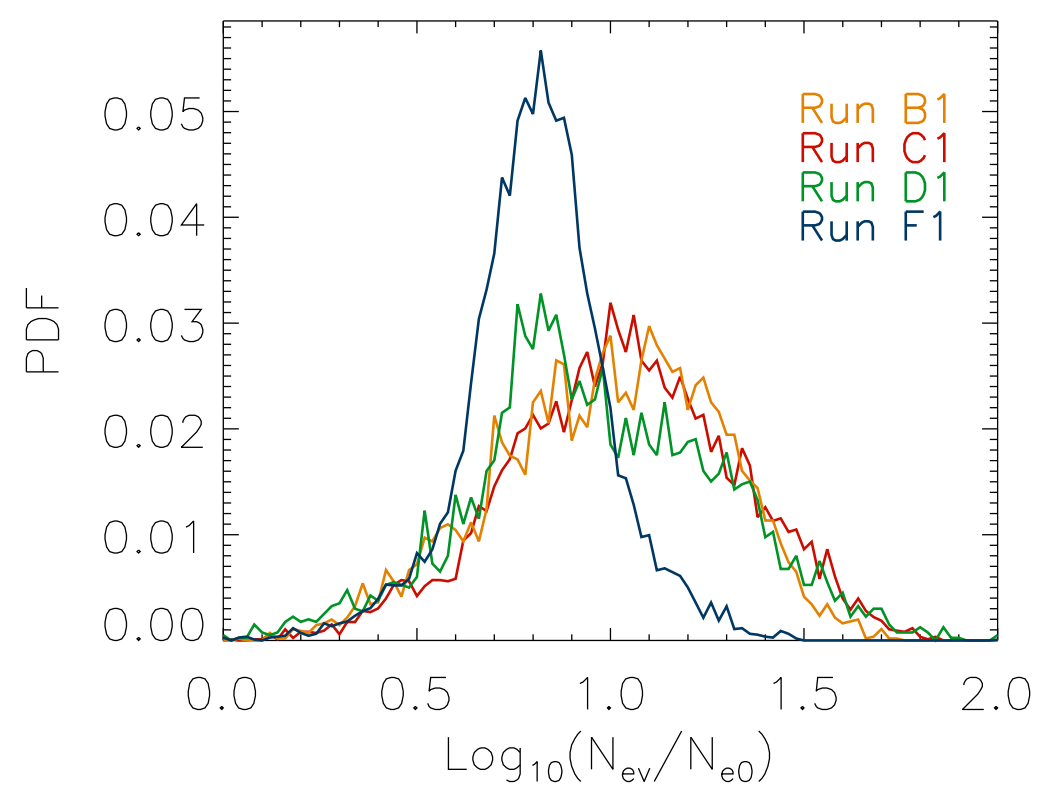

Figure 3: Probability density functions of peak particle density inside magnetic vortices for runs B1 (yellow), C1 (red), D1 (green) and F1 (blue).

Figure 2(a) displays the ramp region for run D1. The foot region with electrostatic Buneman waves is located at $x / \lambda_{s i} \approx(68-72)$ (not shown in Fig. 2(a)) and the overshoot is at $x / \lambda_{s i} \approx 93$. The existence of magnetic islands resulting from reconnection is demonstrated in the enlarged view in Figure 2(b). The density peaks are encircled by magnetic-field lines, which is a characteristic configuration for magnetic reconnection in a Harris current sheet [10]. The magnetic-reconnection events can be identified as chains of magnetic islands separated by X-points, which result from nonlinear decay of the current sheets [11].

The ramp region contains variety of different structures: freshly formed dense filaments are at $x / \lambda_{s i} \approx(75-83)$, dense filaments undergoing magnetic reconnection at $x / \lambda_{s i} \approx(83-87)$, and dense single magnetic islands remaining after magnetic-island coalescence at $x / \lambda_{s i} \approx(87-91)$.

The average density (AD) of magnetic vortices (or islands) found in the shock transition and the vortex generation rate (VGR), defined as the average number of islands observed at the shock region divided by the transverse size of the simulation box, are listed in Table 1. The number of reconnection sites grows linearly with ion-to-electron mass ratio (see runs $\mathrm{A}, \mathrm{B}, \mathrm{C}$ and $\mathrm{D}$ ). The VGR is almost twice larger for runs with $\varphi=45^{\circ}$ than with in-plane magnetic field. The vortex statistics of runs $\mathrm{C}$ and D indicates that for moderate plasma beta (runs C2 and D2) VGR is higher than for cold plasma (runs C2 and D2).

The probability density of peak particle density in magnetic vortices is presented in Figure 3 for 4 runs. Simulations with in-plane magnetic-field (runs A, B, C and D) provide similar probability densities of peak particle density and AD (see Table 1). Slightly higher value of AD for run A1 can be explained by a small vortex statistics. We conclude that the properties of the vortices do not depend on the ion-to-electron mass ratio, while the number of reconnection sites grows. Likewise, the probability density does not depend on the plasma beta.

The probability distribution of particle density in the magnetic islands for run F1 strongly 
differs from that for runs with in-plane magnetic field. Filament decay generates vortices with smaller peak density, and in addition many magnetic vortices arise not from the decay of magnetic filaments but are generated by magnetic turbulence in the ramp region.

\subsection{Acceleration processes related to magnetic reconnection}

It is well known that magnetic reconnection is a process that converts magnetic energy into thermal and kinetic energy. A number of articles are dedicated to the electron energization via magnetic reconnection $[6,12,13]$.

Two sample accelerated particles that reach nonthermal energy in our simulations are presented in Figure 4. The first electron (Fig. 4a) is accelerated by the z-component of the electric field at an X-point. During acceleration the electron moves almost entirely in the z-direction and very little in the $\mathrm{X}-\mathrm{y}$-plane. This is an example of a Speiser motion $[14,15]$. The second electron is captured by a magnetic vortex and accelerated via the "island surfing" mechanism [16].

There are a number of acceleration processes related to magnetic reconnection [12] but here we can identify only a few of them on account of the limited simulation time, whereas dedicated simulations of magnetic reconnection may cover $\sim 100 \Omega_{i}^{-1}[12]$. Thus our simulations describe only the early stage of the decay of a Harris current sheet.

\subsection{Downstream spectra for various mass ratios}

We conclude the presentation of our results with energy spectra of electrons in the downstream region. The spectra are calculated in a region downstream of the overshoot with size corresponding to two cycles of the shock self-reformation. In this way we average over all spectral distorions arising from the shock reformation.
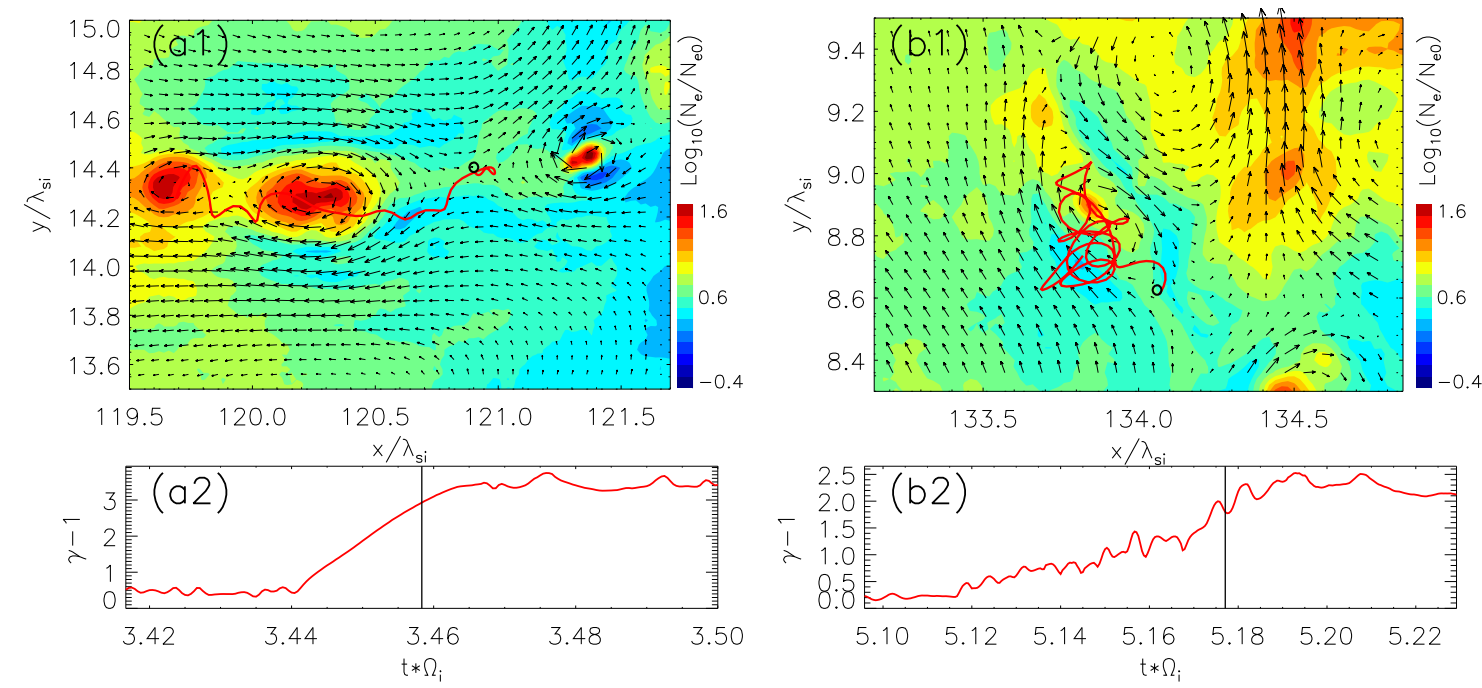

Figure 4: Trajectories of two accelerated electrons for run B2. Top panels (a1 , b1): color - normalized electron density in logarithmic scale; arrows - x-y magnetic-field lines; black circle - position of particle at the specific time marked in the bottom panel; red line - trajectory of said electron over $\Delta t=0.063 \Omega_{i}^{-1}$. Bottom panels (a2, b2): Time evolution of kinetic energy, $\gamma-1$, of the electron. 


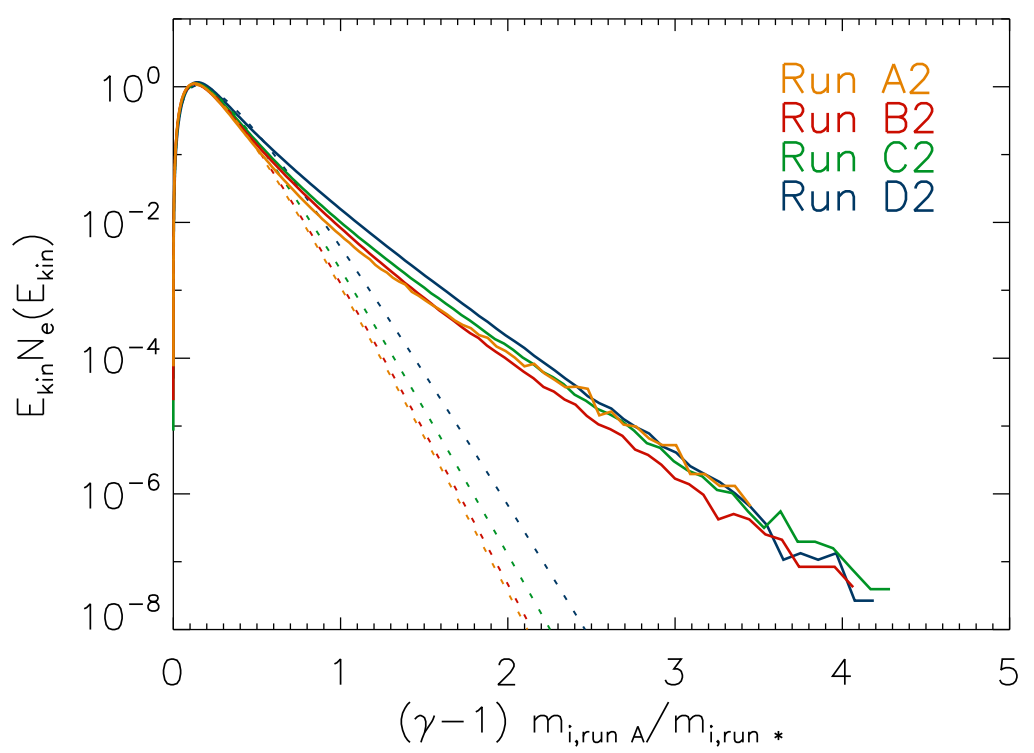

Figure 5: Rescaled downstream spectra for runs A2 (yellow), B2 (red), C2 (green) and D2 (blue). The dashed lines represent fits of a relativistic Maxwellian to the low-energy part of the spectra.

Rescaled spectra for runs with in-plane magnetic-field configuration are presented in Figure 5. The nonthermal electron fractions for runs A, B, C and D listed in Table 1 are the same, and the dotted lines in Figure 5, representing Maxwellian fits to the low-energy spectra, show that the downstream bulk temperature linearly scales with the ion-to-electron mass ratio.

Our previous investigation demonstrates that the nonthermal electron fraction is defined by the intensity of electrostatic Buneman waves. Comparing spectra for various ion-to-electron mass ratios, and hence different abundance of magnetic-reconnection events, we can conclude that magnetic reconnection does not have a significant influence on the downstream electron spectra.

\section{Summary and discussion}

Here we present results of large-scale 2D3V PIC simulations of electron injection at nonrelativistic perpendicular collisionless plasma shocks with high Alfvénic Mach number in whose ramp region a spontaneous magnetic reconnection is observed. Our results can be summarized as follows:

- Collision of two plasma slabs leads to the formation of two shocks propagating in the opposite directions, with the developing time of the shocks of about $2 \Omega^{-1}$. The shock transition is mediated by Weibel-type filamentation instabilities that lead to the development of current filaments, magnetic turbulence, and spontaneous magnetic reconnection.

- The number of magnetic-reconnection sites increases with the ion-to-electron mass ratio. The probability distribution of electron density in magnetic vortices is different for in-plane and for $45^{\circ}$ magnetic-field configurations. 
- Interaction of electrons with magnetic-reconnection sites leads to electron energization up to nonthermal energies. Acceleration occurs in many different ways: capturing of electrons by magnetic islands, acceleration when the particles are placed between colliding magnetic islands, collision of electrons with X-points, and stochastic collisions with magnetic vortices (second-order Fermi-like acceleration).

- Downstream electron spectra for simulations with different mass ratios demonstrate statistically indistinguishable nonthermal fractions. A role of magnetic reconnection in electron injection requires further detailed scrutiny.

\section{Acknowledgments}

The work of A.B., J.N. and O.K. is supported by Narodowe Centrum Nauki through research project DEC-2013/10/E/ST9/00662. M.P. acknowledges support through grants PO 1508/1-1 and PO 1508/1-2 of the Deutsche Forschungsgemeinschaft. Numerical simulations have been performed on the Prometheus system at ACC Cyfronet AGH. Part of the numerical work was also conducted on resources provided by The North-German Supercomputing Alliance (HLRN) under project bbp00003.

\section{References}

[1] Blandford, R., \& Eichler, D. 1987, Physics Reports, 154, 1

[2] Aharonian, F., Akhperjanian, A. G., Bazer-Bachi, A. R., et al. 2007, A\&A, 464, 235

[3] Matsumoto, Y., Amano, T., Kato, T. N., \& Hoshino, M. 2015, Science, 347, 974

[4] Wieland, V., Pohl, M., Niemiec, J., Rafighi, I., \& Nishikawa, K.-I. 2016, ApJ, 820, 62

[5] Matsumoto, Y., Amano, T., \& Hoshino, M. 2012, ApJ, 755, 109

[6] Matsumoto, Y., Amano, T., \& Hoshino, M. 2013, Physical Review Letters, 111, 215003

[7] Buneman, O. 1993, in Computer Space Plasma Physics: Simulation Techniques and Software, Eds.: Matsumoto \& Omura, Tokyo: Terra, p.67

[8] Niemiec, J., Pohl, M., Stroman, T., \& Nishikawa, K.-I. 2008, ApJ, 684, 1174-1189

[9] Kato, T. N., \& Takabe, H. 2010, ApJ, 721, 828

[10] Harris, E. G. 1962, Il Nuovo Cimento, 23, 115

[11] Furth, H. P., Killeen, J., \& Rosenbluth, M. N. 1963, Physics of Fluids, 6, 459

[12] Oka, M., Phan, T.-D., Krucker, S., Fujimoto, M., \& Shinohara, I. 2010, ApJ, 714, 915

[13] Dahlin, J. T., Drake, J. F., \& Swisdak, M. 2014, Physics of Plasmas, 21, 092304

[14] Speiser, T. W. 1965, JGR, 70, 4219

[15] Hoshino, M., Mukai, T., Terasawa, T., \& Shinohara, I. 2001, JGR, 106, 25979

[16] Drake, J. F., Swisdak, M., Che, H., \& Shay, M. A. 2006, Nature, 443, 553 